\title{
A 2D dual-mode composite ultrasonic transducer excited by a single piezoceramic stack
}

Long $\mathrm{Xu}^{\text {a }}$, Xiaojun Qiu ${ }^{\mathrm{b}}$, Jincheng Zhou ${ }^{\text {a }}$, Fengming $\mathrm{Li}^{\text {a }}$, Haidao Zhang a Yuebing Wang ${ }^{\mathrm{a}}$

${ }^{a}$ Department of Physics, China Jiliang University, Hangzhou 310018, China

${ }^{\mathrm{b}}$ Centre for Audio, Acoustics and Vibration, Faculty of Engineering and Information Technology, University of Technology, Sydney, New South Wales 2007, Australia

E-mail: xulong@cjlu.edu.cn

\begin{abstract}
A 2D composite ultrasonic transducer with two resonance modes is proposed by using a central coupling metal block, a piezoceramic stack and four outer metal cylinders. With the introduction of the dual-mode equivalent mechanical coupling coefficient and the 2D longitudinal force transform coefficient, a novel equivalent circuit model is developed for the design and analysis of the 2D coupled vibration of the transducer. After verifying the proposed equivalent circuit model with the FE method and the corresponding experiments, the vibration characteristics of the proposed transducer are investigated by using the equivalent circuit mode, the FE methods, and the experiments. The results demonstrate that the proposed transducer has the advantage of generating 2D ultrasonic radiation with two different fundamental resonance modes, which is useful in some new ultrasonic applications such as ultrasonic emulsification, ultrasonic defoaming and ultrasonic sonochemistry where multi-frequency and multidimensional sound radiation is needed.
\end{abstract}

\section{Introduction}

One dimensional (1D) longitudinal sandwich piezoelectric transducers are widely used for power ultrasonic and underwater SONAR applications thanks to their advantages of high power sound emission, simple structure and high mechanical strength [1-4]. However, in some applications such as large power ultrasonic cleaning, ultrasonic processing, ultrasonic defoaming and ultrasonic motor, transducers with 
larger ultrasonic power, larger radiating surface, multidimensional sound radiation or multi-frequency are needed and novel design theories of these transducers need to be established.

Some measures have been taken to improve the commonly used 1D longitudinal sandwich piezoelectric transducers [5-9]. However, these traditional 1D longitudinal sandwich piezoelectric transducers have some fundamental limitations. First, the 1D longitudinal vibrational theory is used in the design and calculation, which means that the lateral dimension of the sandwich transducer should be less than a quarter wavelength. Consequently, the output power and sound radiation surface of the transducer are limited. Second, the sound radiation for the traditional longitudinal sandwich piezoelectric transducer is one dimensional and this limits the process range of ultrasonic radiation from some ultrasonic applications where large liquid processing volume is needed [10-13].

Meanwhile, 1D longitudinal vibration of the traditional sandwich piezoelectric transducers limits the directions of ultrasonic radiation in some ultrasonic applications where multiple directional sound radiations are needed simultaneously [14-17]. In order to achieve higher electro-acoustic conversion efficiency, the sandwich piezoelectric transducer usually works at a fixed-frequency (commonly the fundamental frequency). However, in the field of ultrasonic chemistry, biomedical ultrasound and ultrasonic waste water treatment, multi-frequency ultrasound has been reported as a promising method to enhance cavitation activities and reduce the cavitation threshold [18-21].

In order to overcome the weakness of the traditional longitudinal sandwich piezoelectric transducers mentioned above, a novel 2D dual-mode composite ultrasonic transducer excited by a single piezoceramic stack is proposed. Based on the 2D coupled vibration theory and the electromechanical analogy principle, the dual-mode equivalent mechanical coupling coefficient and the 2D longitudinal force transform coefficient are introduced first, and then a novel equivalent circuit model is developed to analyze the $2 \mathrm{D}$ coupled vibrations of the transducer. The vibrational characteristics of the transducer are investigated by using the analytical and numerical 
methods, and the experimental measurement. The objective of the research is to provide theoretical and experimental references for the engineering applications of the multi-frequency and multidimensional sound radiation transducers.

\section{Analyses of the proposed transducer}

The proposed 2D dual-mode composite ultrasonic transducer consists of a central coupling metal block, a piezoceramic stack and four outer metal cylinders, which constitute the transducer with a structure of a half-wave sandwich piezoelectric vibrator in its $\mathrm{y}$ direction and a half-wave metal vibrator in its $\mathrm{x}$ direction. Both half-wave vibrators are coupled orthogonally with each other at the oscillation node of their longitudinal vibrations as shown in Figure 1. After optimizing the dimensions for each component of the transducer, two different longitudinal orthogonal resonance modes at certain frequencies can be realized. One is the anti-phase resonance mode generated by the half-wave sandwich piezoelectric vibrator that extends along its axial direction and the half-wave metal vibrator that shortens along its axial direction at the same time, and vice versa. The other is the in-phase resonance mode which extends or shortens along their axial directions at the same time.

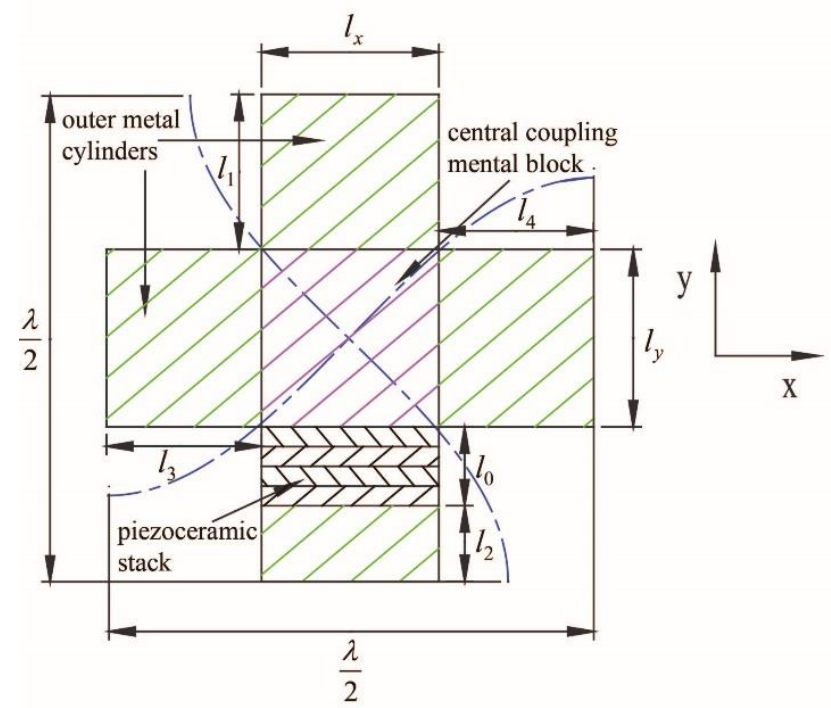

(a)

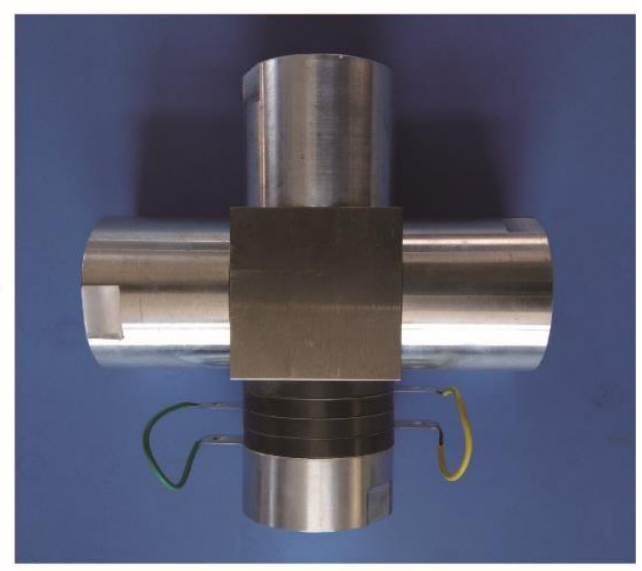

(b)

Figure 1. The 2D dual-mode composite ultrasonic transducer excited by a single piezoceramic stack (a) diagram (b) a photo. 


\subsection{Equivalent circuit of the central coupling metal block in coupled vibration}

As shown in figure 1(a), the length, width and height of the central coupling metal block are $l_{x}, l_{y}$ and $l_{z}$ respectively, where $l_{x}=l_{y}=l_{z}=l, l_{3}$ and $l_{4}$ are the length of the left and right metal cylinders of the transducer in $\mathrm{x}$ direction, $l_{1}$ and $l_{2}$ are the length of the up and down outer metal cylinders in the y direction, the piezoceramic stack is composed of two couples of piezoelectric rings with radius $r$ and thickness $t$, and the total length of the piezoelectric stack is $l_{0}=n t$ with $n=4$ in this design.

For the anti-phase vibration and in-phase vibration of the transducer, when its shearing strain and torsion are ignored, i.e. only its extensional vibration is considered, the following equations between the stress and strain in the central coupling metal block of the transducer can be obtained [22,23].

$$
\begin{aligned}
& \varepsilon_{x}=\frac{1}{E}\left[\sigma_{x}-v\left(\sigma_{y}+\sigma_{z}\right)\right] \\
& \varepsilon_{y}=\frac{1}{E}\left[\sigma_{y}-v\left(\sigma_{x}+\sigma_{z}\right)\right] \\
& \varepsilon_{z}=\frac{1}{E}\left[\sigma_{z}-v\left(\sigma_{x}+\sigma_{y}\right)\right]
\end{aligned}
$$

where $\varepsilon_{x}, \varepsilon_{y}, \varepsilon_{z}$ and $\sigma_{x}, \sigma_{y}, \sigma_{z}$ are the strains and stresses, $E$ and $v$ are the Young's modulus and the Poisson ratio of the steel which is used to make the metal block.

This system has two resonance frequencies depending on the vibration phase of the half-wave single-excited sandwich piezoelectric vibrator (in the y direction) and the half-wave metal rod (in the $\mathrm{x}$ direction). Whether in the in-phase or the anti-phase mode, when the dimension of the $2 \mathrm{D}$ dual-mode composite ultrasonic transducer in the $\mathrm{z}$ direction is less than a quarter of the longitudinal wavelength, the strain in $\mathrm{z}$ direction is little and can be ignored [22]. So, $\varepsilon_{z}=0$, and equation (3) can be rewritten as

$$
\sigma_{z}=v\left(\sigma_{x}+\sigma_{y}\right)
$$


For the vibrations in the $\mathrm{x}$ and $\mathrm{y}$ directions of the transducer, to describe their interrelation with each other, the following dual-mode equivalent mechanical coupling coefficient is defined as

$$
n^{\prime}=\frac{\sigma_{x}}{\sigma_{y}}
$$

When $l_{x}=l_{y}$, the central coupling mental block of the transducer has a symmetrical structure in the $\mathrm{x}$ and $\mathrm{y}$ directions. In the anti-phase mode, $\sigma_{x}=-\sigma_{y}$ due to the symmetry of the structure and the anti-symmetry of the vibration mode in the $\mathrm{x}$ and $\mathrm{y}$ directions, so $n^{\prime}=\sigma_{x} / \sigma_{y}=-1$. Similarly, in the in-phase mode, $\sigma_{x}=\sigma_{y}$ duo to the symmetry of both the structure and the vibration mode in the $\mathrm{x}$ and y directions, so $n$ $=\sigma_{x} / \sigma_{y}=1$.

Define $E_{x}=\sigma_{x} / \varepsilon_{x}$ and $E_{y}=\sigma_{y} / \varepsilon_{y}$ as the equivalent elastic constants in the $\mathrm{x}$ and $\mathrm{y}$ directions, respectively, and the equivalent elastic constants in the anti-phase mode and in-phase mode can be rewritten as

$$
\begin{gathered}
E_{x}=E_{y}=E_{-}=\frac{E}{1+v} \\
E_{x}=E_{y}=E_{+}=\frac{E}{1-v-2 v^{2}}
\end{gathered}
$$

In order to establish the equivalent circuit for the coupled vibration of the center coupling metal block in the $\mathrm{x}$ and $\mathrm{y}$ directions, it is assumed that these two directional coupled vibrations consist of 1D longitudinal vibrations of two slender blocks, which coupled orthogonally with each other at the oscillation node of their longitudinal vibration. Based on this assumption and the electromechanical analogy principle, the 1D longitudinal vibration of the slender block in the $\mathrm{x}$ direction is represented by two T-type networks connected in parallel. Similarly, the 1D longitudinal vibration of the slender block in the y direction is represented by two T-type networks connected in parallel. These two parts from the $\mathrm{x}$ and $\mathrm{y}$ directions are coupled at the oscillation node of their longitudinal vibrations with the relationship of each other by a 2D longitudinal force transform coefficient $N$, as shown in Figure 2. $N$ is defined as 


$$
N=\frac{F_{x}}{F_{y}}=\frac{\sigma_{x} S_{x}}{\sigma_{y} S_{y}}=n^{\prime} \frac{S_{x}}{S_{y}}
$$

where $F_{x}$ and $F_{y}$ are the longitudinal forces in the $\mathrm{x}$ and y directions, respectively, $S_{x}$ $S_{x}$ and $S_{y}$ are the cross-sectional areas in the x direction and y direction of the central coupling metal block $\left(S_{x}=S_{y}=S=l^{2}\right) . Z_{1 \mathrm{~m}}=\mathrm{j} Z_{01} \tan \left(k^{\prime} l / 4\right)$ and $Z_{2 \mathrm{~m}}=Z_{01} /\left(\mathrm{j} \sin \left(k^{\prime} l / 2\right)\right)$ are the mechanical impedance parameters, while $Z_{01}=\rho C^{\prime} S, k^{\prime}=\omega / C^{\prime}, k^{\prime}$ is the coupled wavenumber, $\rho$ is the density, $C^{\prime}$ is the coupled sound speed with $C^{\prime}=(E-l \rho)^{-1 / 2}$ in the anti-phase mode and $C^{\prime}=\left(E_{+} / \rho\right)^{-1 / 2}$ in the in-phase mode, respectively.

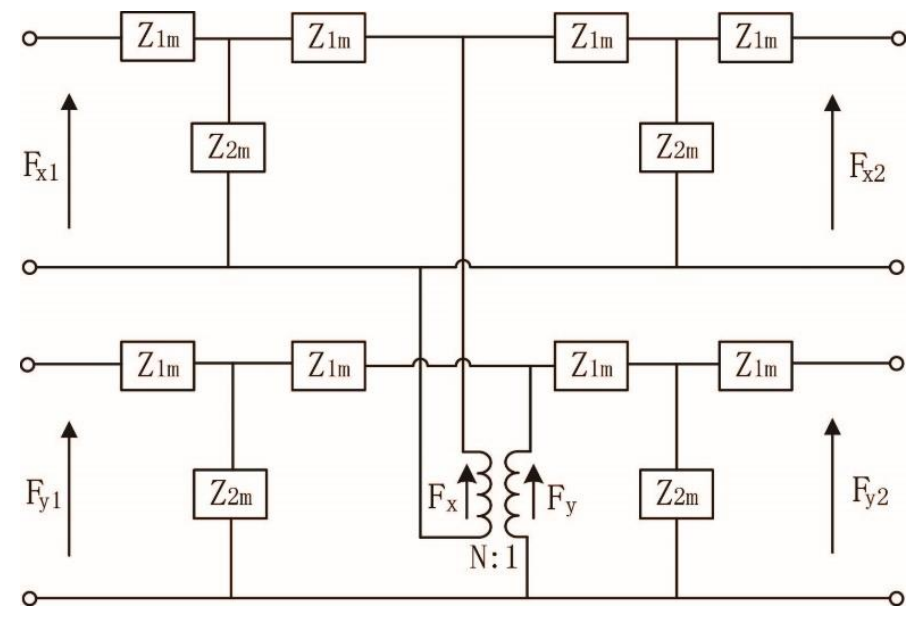

Figure 2. The equivalent circuit for the central coupling metal block in coupled vibration.

\subsection{Equivalent circuit of the outer metal cylinders}

Whether the 2D dual-mode composite ultrasonic transducer vibrates in the anti-phase resonance mode or in the in-phase resonance mode, the four outer metal cylinders of the transducer vibrate in 1D longitudinal vibration. For a $1 \mathrm{D}$ longitudinal vibrational metal cylinder, its equivalent circuit is modeled using a T-network [24], as shown in Figure 3. 


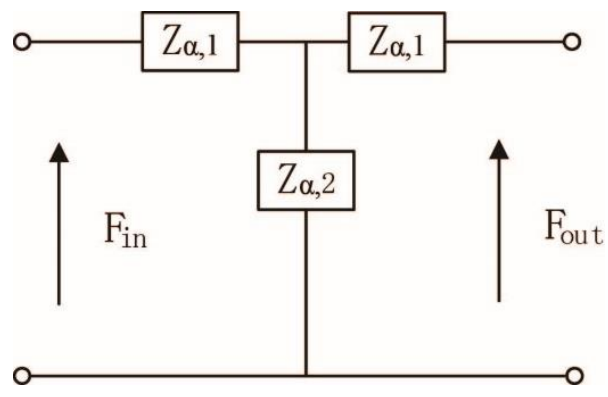

Figure 3. The equivalent circuit of the outer metal cylinder.

In the figure, $Z_{\alpha, 1}=\mathrm{j} Z_{\alpha, 0} \tan \left(k_{\alpha} l_{\alpha} / 2\right)$ and $Z_{\alpha, 2}=Z_{\alpha, 0} / \mathrm{j} \sin \left(k_{\alpha} l_{\alpha}\right)$ are the series and parallel impedances of T-network. The subscript $\alpha=1,2,3,4$ corresponds to different outer metal cylinder (as shown in Figure 1), where $Z_{\alpha, 0}=\rho_{\alpha} c_{\alpha} S_{\alpha}, \rho_{\alpha}, c_{\alpha}$ and $S_{\alpha}$ are the density, velocity and cross-sectional area of the different outer metal cylinder. When the proposed transducer vibrates freely, the external loads of the four outer metal cylinders are ignored. Therefore, the equivalent circuit for each outer metal cylinder of the transducer in Figure 3 can be simplified as shown in Figure 4, where $Z_{\alpha}=Z_{\alpha, 1}+$ $Z_{\alpha, 1} Z_{\alpha, 2} /\left(Z_{\alpha, 1}+Z_{\alpha, 2}\right)$.

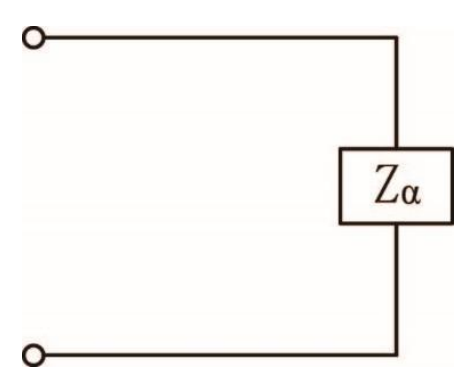

Figure 4. The simplified equivalent circuit for the outer metal cylinder.

\subsection{Equivalent circuit of the piezoceramic stack}

For a piezoceramic stack, it is usually modeled using the Mason's equivalent circuit [7,24,25]. As shown in Figure 5, the parameters of the equivalent circuit for the piezoceramic stack are: $C_{0}=n^{2} \varepsilon_{33}^{T} S_{0}\left(1-k_{33}^{2}\right) / l_{0}, N_{1}=n S_{0} d_{33} /\left(l_{0} S_{33}^{E}\right), Z_{1 \mathrm{P}}=\mathrm{j} Z_{0 \mathrm{P}} \tan \left(k_{\mathrm{p}} l_{0} / 2\right)$, $Z_{2 \mathrm{P}}=Z_{0 \mathrm{P}} / \mathrm{j} \sin \left(k_{\mathrm{p}} l_{0}\right)$. 


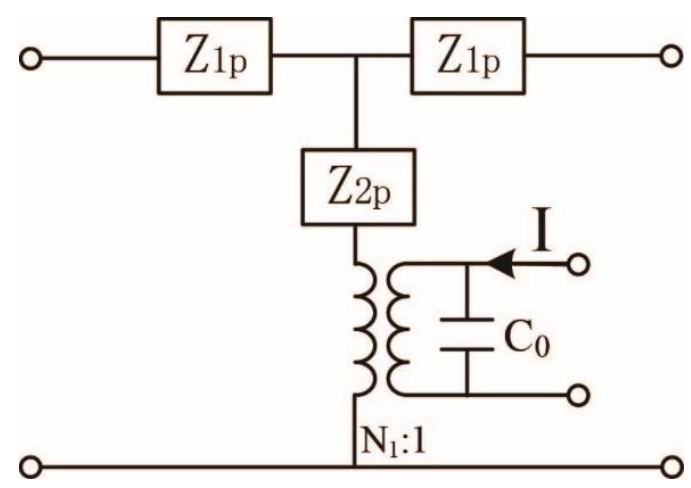

Figure 5. The equivalent circuit for a piezoceramic stack.

\subsection{Equivalent circuit of the whole transducer}

Based on the electromechanical analogy principle, using the continuous boundary conditions of the longitudinal force and velocity at the interface of each component of the transducer shown in Figure 1(a), the integral equivalent circuit of the transducer can be obtained from Figures 2, 4 and 5, which is shown in Figure 6, where $Z_{1}, Z_{2}, Z_{3}$ and $Z_{4}$ correspond to the different $Z_{\alpha}(\alpha=1,2,3,4)$, and $Z_{\alpha}=Z_{\alpha, 1}+Z_{\alpha, 1} Z_{\alpha, 2} /\left(Z_{\alpha, 1}+Z_{\alpha, 2}\right)$ shown in Figure 4.

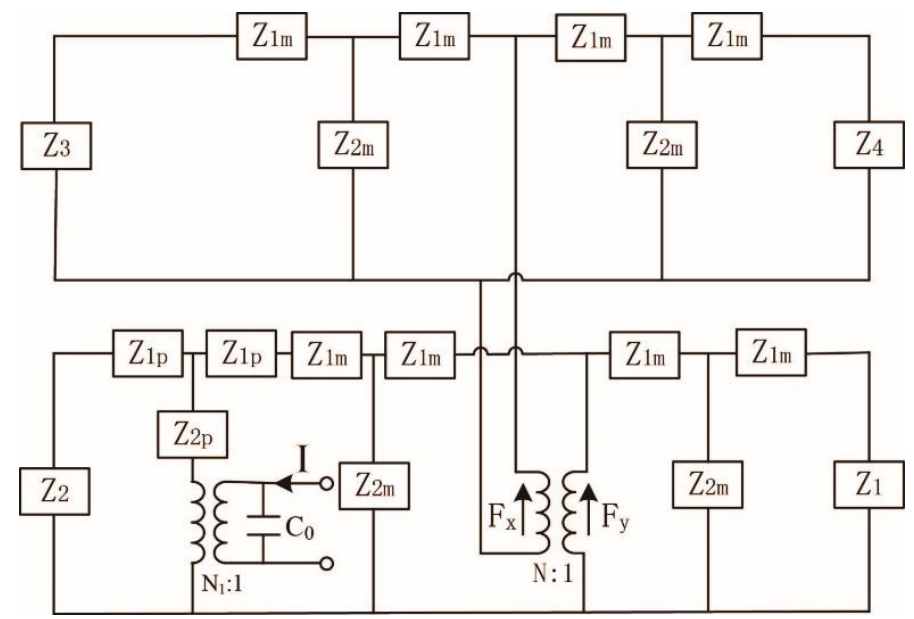

Figure 6. The equivalent circuit for the $2 \mathrm{D}$ dual-mode composite ultrasonic transducer.

Based on Figure 6, using the transmission line impedance transfer formula, the input electro-mechanical impedance of the transducer can be obtained as 


$$
Z_{e m}=\frac{Z_{m}}{N_{1}^{2}+j \omega C_{0} Z_{m}}
$$

where $Z_{m}$ is the input mechanical impedance of the transducer, which can be expressed as

$$
Z_{m}=Z_{2 p}+\frac{\left(Z_{2}+Z_{1 p}\right) \cdot\left(Z_{x y}+Z_{1 p}\right)}{2 Z_{1 p}+Z_{2}+Z_{x y}}
$$

with

$$
\begin{gathered}
Z_{x y}=Z_{1 m}+\frac{\left(Z_{1 y}+Z_{1 m}\right) \cdot Z_{2 m}}{Z_{1 y}+Z_{1 m}+Z_{2 m}} \\
Z_{1 y}=\frac{N^{2} \cdot Z_{y} \cdot Z_{x m 1}}{N^{2} \cdot Z_{y}+Z_{x m 1}} \\
Z_{y}=\frac{Z_{y m 3} \cdot Z_{y m 4}}{Z_{y m 3}+Z_{y m 4}} \\
Z_{x m 1}=Z_{1 m}+\frac{\left(Z_{1}+Z_{1 m}\right) \cdot Z_{2 m}}{Z_{1}+Z_{1 m}+Z_{2 m}} \\
Z_{y m 3}=Z_{1 m}+\frac{\left(Z_{3}+Z_{1 m}\right) \cdot Z_{2 m}}{Z_{3}+Z_{1 m}+Z_{2 m}} \\
Z_{y m 4}=Z_{1 m}+\frac{\left(Z_{4}+Z_{1 m}\right) \cdot Z_{2 m}}{Z_{4}+Z_{1 m}+Z_{2 m}}
\end{gathered}
$$

The resonance frequency equation for the transducer can be obtained by setting

$$
\operatorname{Im}\left(Z_{e m}\right)=0
$$

It is clear that the resonance frequency equation for the transducer depends not only on the material parameters and geometrical dimensions, but also on the resonance modes of the transducer. When the vibration mode $\left(n^{\prime}=-1\right.$ or $\left.n^{\prime}=1\right)$, the material parameters and geometrical dimensions of the transducer are given, the resonance frequency can be calculated from the resonance frequency equation. Furthermore, the theoretical relationship between the input mechanical reactance $X_{e m}$ $=\operatorname{Im}\left(Z_{e m}\right)$ and the frequency $f$ can also be obtained. 


\section{Vibration characteristics of the transducer}

In order to verify the proposed equivalent circuit model and to understand the vibrational characteristics of the proposed transducer, a prototype transducer was designed, which is shown in Figure 1(b). The geometrical dimensions of the transducer shown in Figure 1(a) are: the length, width and height of the central coupling metal block $l_{x}=l_{y}=l_{z}=45.0 \mathrm{~mm}$, the length of the up and down outer metal cylinders in the y direction $l_{1}=38.3 \mathrm{~mm}, l_{2}=19.3 \mathrm{~mm}$, length of the left and right metal cylinders of the transducer in $\mathrm{x}$ direction $l_{3}=l_{4}=38.3 \mathrm{~mm}$, the length of the piezoelectric stack $l_{0}=20 \mathrm{~mm}$, and the radius of the piezoelectric stack and the metal cylinders $r=22.5 \mathrm{~mm}$. The material properties for each part of the transducer are as follows. The material properties for central coupling metal block (stainless steel) are: density $\rho=7910 \mathrm{~kg} / \mathrm{m}^{3}$, Young's modulus $E=1.96 \times 10^{11} \mathrm{~N} / \mathrm{m}^{2}$, Poisson ratio $\sigma=0.30$. The material properties for four outer metal cylinders (7075 aluminum) are: density $\rho_{\alpha}=2790 \mathrm{~kg} / \mathrm{m}^{3}$, Young's modulus $E_{\alpha}=7.15 \times 10^{10} \mathrm{~N} / \mathrm{m}^{2}$, Poisson ratio $\sigma_{\alpha}=0.30$, and the material properties for piezoceramic stack (PZT-4) are listed as Table 1.

Table 1. The material parameters of PZT-4 used for the transducer.

\begin{tabular}{llll}
\hline & Constant & Units & Value \\
\hline Elastic compliance constants & $S_{11}^{E}$ & $\mathrm{~m}^{2} \cdot \mathrm{N}^{-1}$ & $12.3 \times 10^{-12}$ \\
Elastic compliance constants & $S_{12}^{E}$ & $\mathrm{~m}^{2} \cdot \mathrm{N}^{-1}$ & $-4.05 \times 10^{-12}$ \\
Elastic compliance constants & $S_{13}^{E}$ & $\mathrm{~m}^{2} \cdot \mathrm{N}^{-1}$ & $-5.31 \times 10^{-12}$ \\
\hline Elastic compliance constants & $S_{33}^{E}$ & $\mathrm{~m}^{2} \cdot \mathrm{N}^{-1}$ & $15.5 \times 10^{-12}$ \\
Piezoelectric strain constants & $d_{31}$ & $\mathrm{C} \cdot \mathrm{N}^{-1}$ & $-123 \times 10^{-12}$ \\
Piezoelectric strain constants & $d_{33}$ & $\mathrm{C} \cdot \mathrm{N}^{-1}$ & $496 \times 10^{-12}$ \\
\hline Dielectric constant & $\varepsilon_{33}^{T}$ & $\mathrm{~F} \cdot \mathrm{m}^{-1}$ & $11.5 \times 10^{-9}$ \\
\hline Electro-mechanical coupling coefficient & $k_{33}$ & & 0.70 \\
\hline Density & $\rho_{p}$ & $\mathrm{~kg} \cdot \mathrm{m}^{-3}$ & 7500 \\
\hline
\end{tabular}

\subsection{The input electromechanical impedance-frequency response}


The frequency resonance characteristics can be obtained by using the equivalent circuit method (ECM), the FEM and experiment. First, from equation (9), the input electro-mechanical reactance spectrum of the transducer was calculated by the ECM, which are illustrated in Figure 7, where the first resonance peak (red dash curve) corresponds to the anti-phase resonance peak and the second resonance peak (green dash curve) corresponding to the in-phase resonance peak. Then, the input electro-mechanical reactance spectrum was simulated by the Harmonic Analysis from the FE software ANSYS, as is shown in black curve in Figure 7, where the first resonance peak corresponds to the anti-phase resonance peak and the second resonance peak corresponding to the in-phase resonance peak.

The input electro-mechanical reactance spectrum was also measured by using a PV70 Impedance Analyzer, as is shown in blue shot dot curve in Figure 7. It can be seen that two resonance peaks exist in the measured frequency range, which correspond to the anti-phase resonance mode and the in-phase resonance mode, respectively. The resonance frequencies for the anti-phase resonance mode obtained by the ECM, FEM and the experiment are $24071 \mathrm{~Hz}, 23452 \mathrm{~Hz}$, and $23290 \mathrm{~Hz}$, respectively, while the resonance frequencies for the in-phase resonance mode obtained by the three methods are $27933 \mathrm{~Hz}, 27716 \mathrm{~Hz}, 27635 \mathrm{~Hz}$, respectively. It is clear that the anti-phase and in-phase resonance frequencies measured by the experiments are in consistent with that obtained by the ECM and FE methods. 


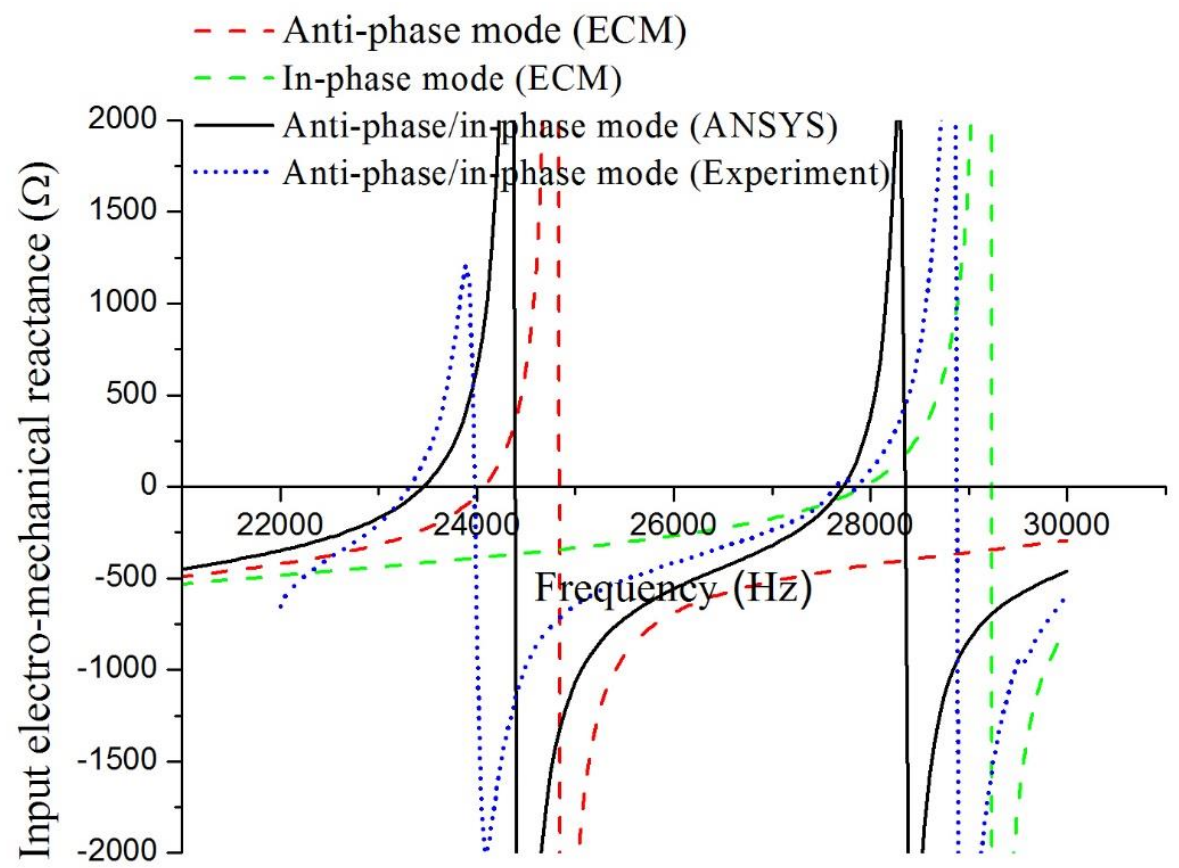

Figure 7. Input electro-mechanical reactance spectrum of the transducer in the anti-phase and in-phase modes calculated by the ECM, FEM and experiment, respectively.

\subsection{Investigation of the anti-phase and in-phase resonance modes}

The modal analysis results obtained with the FE software ANSYS are illustrated in Figure 8, where Figure 8(a) shows that the vibration phases in the $\mathrm{x}$ and $\mathrm{y}$ directions (horizontal and vertical directions) are opposite, which is the anti-phase resonance mode, while Figure 8(b) shows that the vibration phases in the $\mathrm{x}$ and $\mathrm{y}$ directions are consistent, which is the in-phase resonance mode. The simulated vibration frequency in the anti-phase mode is $f=23452 \mathrm{~Hz}$ and that in the in-phase mode is $f=27716 \mathrm{~Hz}$. Figure 9 shows the longitudinal vibrational displacement distributions along the axis of the half-wave sandwich piezoelectric vibrator in the $\mathrm{x}$ direction of the transducer and that of the half-wave metal vibrator in the y direction, respectively, where it is clear that whether it operates in the anti-phase mode or the in-phase mode, the transducer is composited by two half-wave oscillators, and both are coupled orthogonally together at the oscillation node of their longitudinal vibrations. 


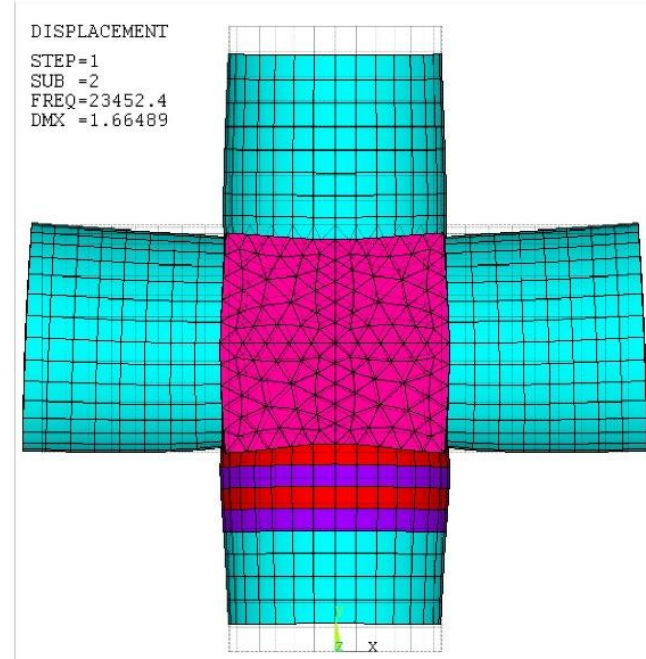

(a)

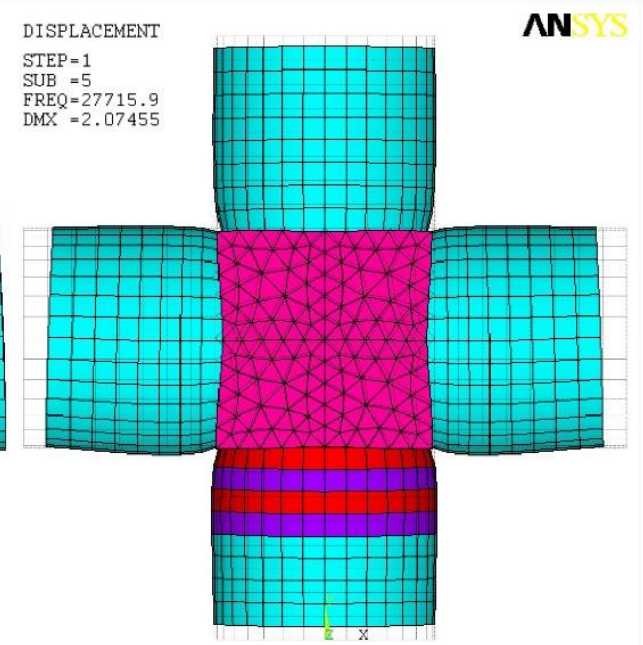

(b)

Figure 8. Modal shape of the transducer (a) in the anti-phase mode at $23452 \mathrm{~Hz}$ and (b) in the in-phase mode at $27716 \mathrm{~Hz}$.

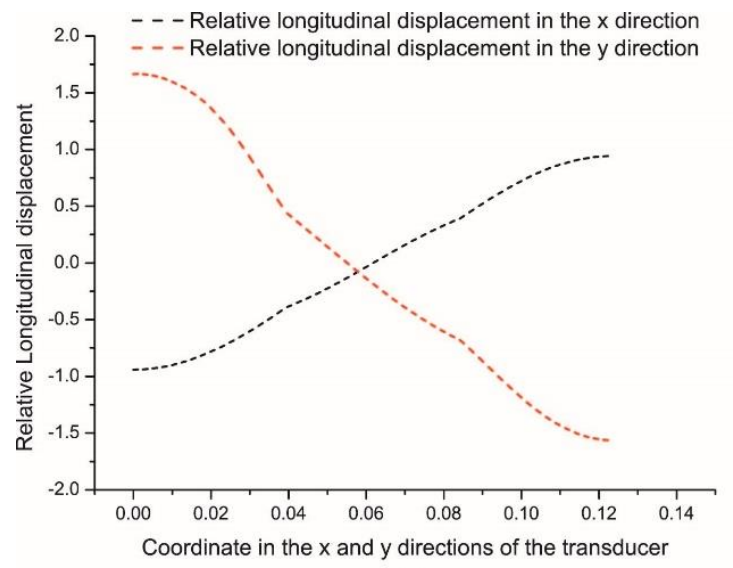

(a)

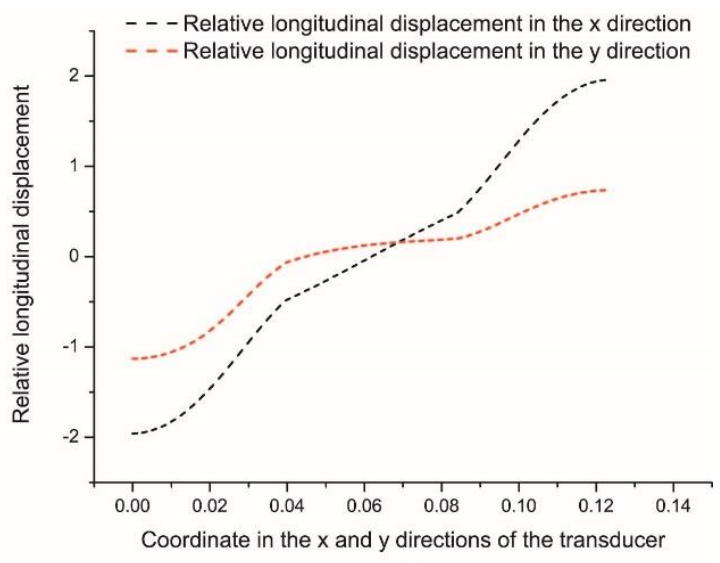

(b)

Figure 9. Longitudinal vibrational displacement distributions for the transducer along its $\mathrm{x}$ and $\mathrm{y}$ directions (a) in the anti-phase mode and (b) in the in-phase mode.

The vibration characteristics of the prototype transducer in the anti-phase and in-phase modes were measured by using a Polytec PSV-400 Scanning Vibrometer. Whether the proposed transducer vibrates in the anti-phase resonance mode or in the in-phase resonance mode, the vibration in its output surfaces from the $\mathrm{x}$ and $\mathrm{y}$ directions should be similar as a piston. In the experiment, when the transducer was excited by a $10 \mathrm{~V}$ sinusoidal sweep signal provided by the Vibrometer, the laser 
sensor scanned on the output surfaces of the transducer. Then, the longitudinal displacement field of the transducer can be obtained.

Figure 10(a) shows the longitudinal displacement field of the output surface in the $\mathrm{x}$ direction of the transducer vibrated in the anti-phase resonance mode, while Figure 10(b) shows that in the y direction. Figure10(c) shows the longitudinal displacement field of the output surface in the $\mathrm{x}$ direction of the transducer vibrated in the in-phase resonance mode, and Figure 10(d) shows that in the y direction. In both modes, the vibration of the output surfaces in both $\mathrm{x}$ and $\mathrm{y}$ directions for the transducer vibrates like a piston; however, the longitudinal displacement fields in the y direction are more uniform than that in the $\mathrm{x}$ direction. This is because that the both modes are excited by the piezoceramic stack in its y direction, which produced little bending deformation in the longitudinal vibration of the $\mathrm{x}$ direction. This phenomenon is also evident from the FE simulated results in Figure 8.

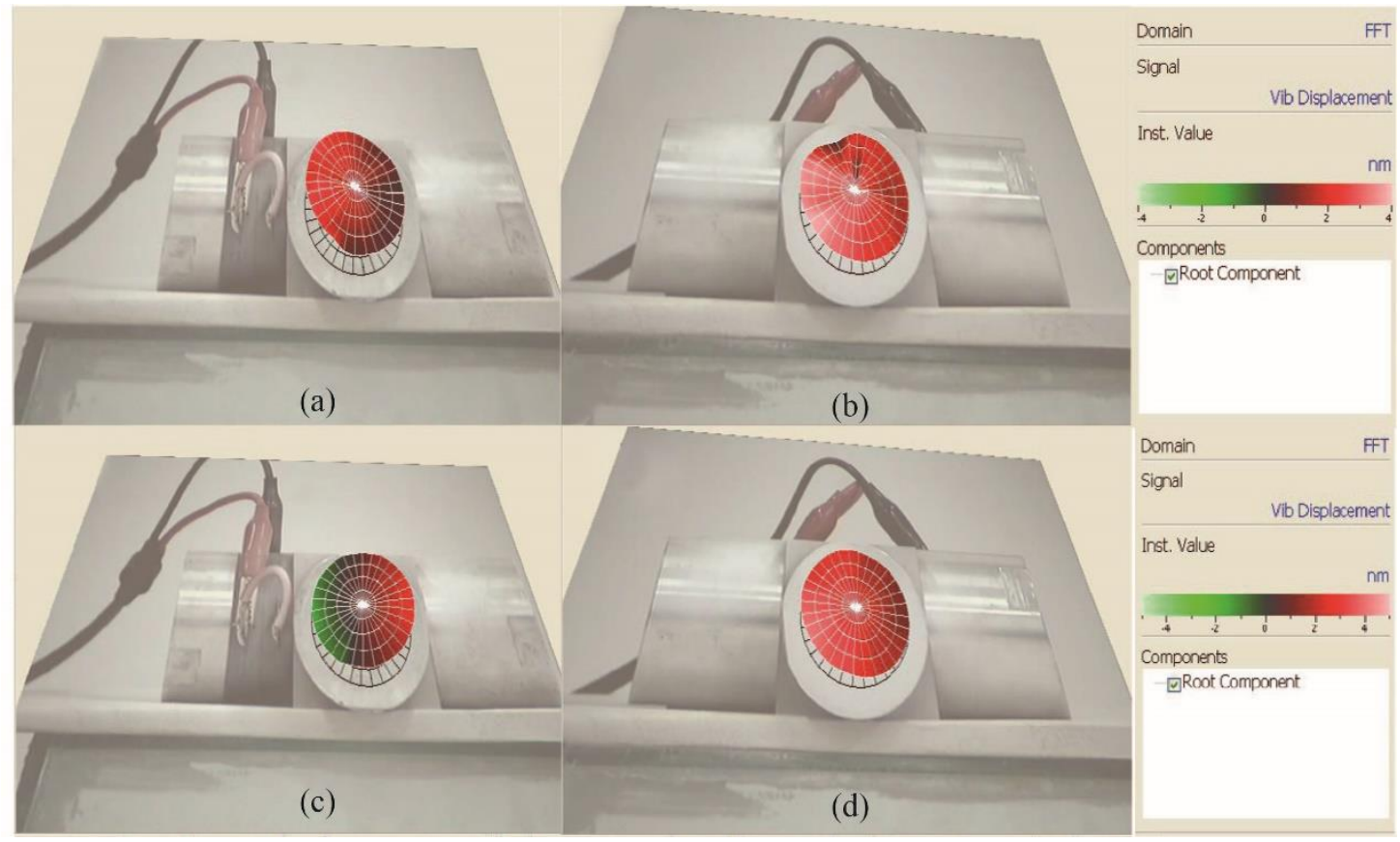

Figure 10. The longitudinal displacement fields of the output surface of the transducer measured by a PSV-400 Scanning Vibrometer (a) in the x direction in the anti-phase mode (b) in the y direction in the anti-phase mode (c) in the x direction in the in-phase mode (d) in the y direction in the in-phase mode. 


\subsection{Experimental test on the performance of the $2 D$ ultrasound radiation}

The performance of the 2D ultrasound radiation of the transducer are tested nearing the anti-phase and in-phase resonance frequencies, respectively. In the experiment, an AG 1020 Amplifier of T\&C power conversion, Inc. is used to amplify the output power of the function generator. The transducer is excited to radiate ultrasound into the oil-water mixture. By adjusting the frequency of the function generator, the input power and the matching resistor of the AG 1020 Amplifier, the transducer can be tuned to the two desired resonance modes, the anti-phase resonance mode (at 22.16 $\mathrm{kHz}$ ) and the in-phase resonance mode (at $27.90 \mathrm{kHz}$ ), and emulsification under the conditions of ultrasonic irradiation from the $\mathrm{x}$ direction and the $\mathrm{y}$ direction of the transducer occurs in these two modes.

Figure 11 shows the photographs of the oil-water mixture before and after ultrasonic emulsification. It can be seen clearly that the obvious ultrasonic emulsification of oil-water mixture under the conditions of ultrasonic irradiation from the $\mathrm{x}$ direction and the $\mathrm{y}$ direction of the transducer occurs. Therefore, the transducer can radiate $2 \mathrm{D}$ intense ultrasound in the anti-phase and in-phase modes. This is different from the case of the traditional 1D longitudinal transducer where ultrasonic irradiation is produced mainly in the longitudinal direction. 


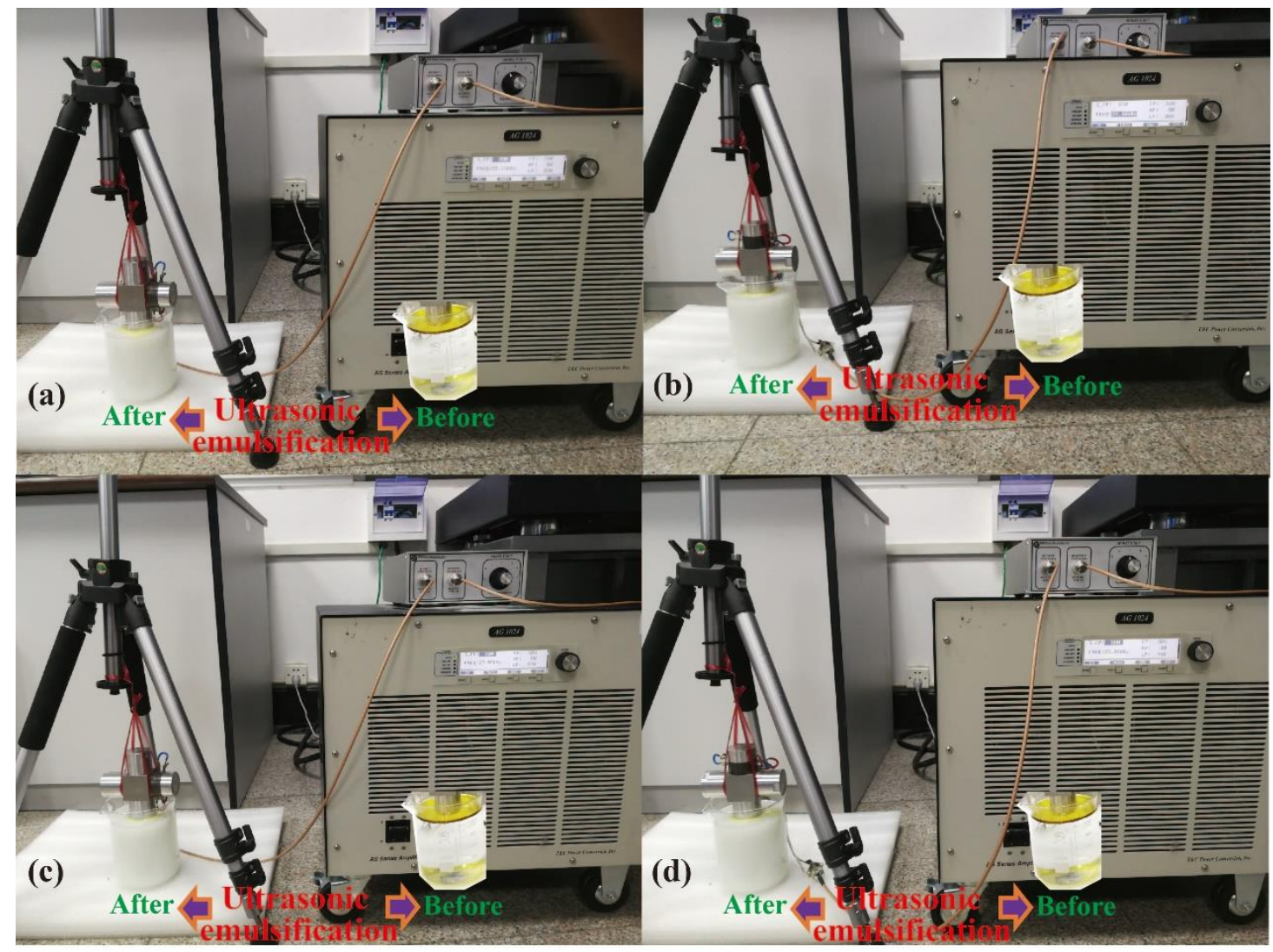

Figure 11. Photographs of the oil-water mixture before and after ultrasonic emulsification, (a) ultrasonic irradiation from the $\mathrm{x}$ direction in anti-phase mode, (b) ultrasonic irradiation from the $\mathrm{y}$ direction in anti-phase mode, (c) ultrasonic irradiation from the $\mathrm{x}$ direction in in-phase mode, (d) ultrasonic irradiation from the $\mathrm{y}$ direction in in-phase mode.

In summary, the proposed transducer has two longitudinal coupled vibration modes for exciting two-dimensional ultrasonic vibration. It is useful in some new ultrasonic applications where multi-frequency or multi-dimensional ultrasound radiation are needed. The experimental results are in reasonable agreement with the analytical and simulated results. The differences between the results might be due to the following factors. First, the standard material parameters for the proposed transducer were used in the theoretical calculation, which might be different from the real material parameters. Second, in the theoretical analysis, the mechanical and dielectric losses in the transducer were ignored. However, there are losses in the prototype transducer used in the experiments, which is difficult to be determined 
accurately. Finally, the prestress is ignored in the theoretical analysis and numerical simulations, but the prototype transducer is prestressed by the stress bolt.

\section{Summary}

In this paper, a 2D dual-mode composite ultrasonic transducer is proposed. A novel equivalent circuit model has been developed to analyze the $2 \mathrm{D}$ coupled vibrations of the transducer in the two different resonance modes. The vibration characteristics of the transducer have been investigated by using the equivalent circuit model, the FE methods, and the experiments. The results demonstrate that the transducer has the advantages of two fundamental resonance modes and 2D ultrasonic radiations. It is expected to be used in some ultrasonic applications where the multi-frequency and multidimensional sound radiation are needed, such as ultrasonic emulsification, ultrasonic defoaming and ultrasonic sonochemistry.

\section{Acknowledgements}

The author acknowledges the National Natural Science Foundation of China (Project nos. 11574277 and 11474259) and the Zhejiang Provincial Natural Science Foundation of China (Project no. LY16A040006) for their financial support.

\section{References}

[1] Heikkola E and Laitinen M, 2005 Model-based optimization of ultrasonic transducers, Ultrason. Sonochem. 12 53-7

[2] Neppiras E A 1973 The pre-stressed piezoelectric sandwich transducer, Ultrasonics International 1973 Conference Proceedings p. 295

[3] Lin S, Tian H 2008 Study on the sandwich piezoelectric ceramic ultrasonic transducer in thickness vibration Smart Mater. Struct. 17015034

[4] Iula A, Vazquez F, Pappalardo M and Gallego J, 2002 Finite element three-dimension analysis of the vibrational behaviour of the Langevin-type transducer Ultrasonics $\mathbf{4 0} 513-7$ 
[5] Ensminger D and Stulen F B 2008 Ultrasonics Data, Equations, and Their Practical Uses (New York: CRC Press)

[6] Ensminger D and Bond L J 2012 Ultrasonics Fundamentals, Technologies, and Applications (New York: CRC Press)

[7] Lin S, Xu L and $\mathrm{Hu}$ W 2011 A new type of high power composite ultrasonic transducer J. Sound Vib. 330 1419-31

[8] $\mathrm{Xu} \mathrm{L}$ and Lin S 2011 Optimization design of high power ultrasonic circular ring radiator in coupled vibration Ultrasonics $\mathbf{5 1} 815-23$

[9] Lin S 1995 Study on the multifrequency Langevin ultrasonic transducer Ultrasonics 33 445-8

[10] Peshkovsky S L and Peshkovsky A S 2007 Matching a transducer to water at cavitation: Acoustic horn design principles Ultrason. Sonochem 14 314-22

[11] Dahlem O, Reisse J and Halloin V 1999 The radially vibrating horn: a scaling-up possibility for sonochemical reactions Chem. Eng. Sci. 54 2829-38

[12] Khmelev V N, Levin S V and Tsyganok S N, 2009 Development and application of piezoelectric transducer with the enlarged radiation surface for wastewater treatment 10th international conference and seminar EDM'2009 section IV pp 254-6

[13] Liang Z, Zhou G, Zhang Y, Li Zand Lin S 2006 Vibration analysis and sound field characteristics of a tubular ultrasonic radiator Ultrasonics 45 146-51

[14] Itoh K, Mori E, 1972 Study on resonator with directional converter(L-L type converter) J. Acoust. Soc. Japan. 28 127-35

[15] Tadeusz G, Lukasz P P and Krzysztof J O 2013 Ultrasonic transducers with directional converters of vibration of longitudinal-longitudinal (L-L) type and longitudinal-longitudinal-longitudinal (L-L-L) type intended to work in gaseous media Proceedings of Meetings on Acoustics 19 pp 1-6

[16] Gudra T 2000 Analysis of a resonator with a directional ultrasonic vibration converter of R-L type using the finite element method Archives of acoustics $\mathbf{2 5}$ $157-74$

[17] Tsujino $\mathrm{J}$ et al 2004 Configurations of high-frequency ultrasonics of complex 
vibration systems for packaging in microelectronics Ultrasonics 42 125-9

[18] Feng R et al 2002 Enhancement of ultrasonic cavitation yield by multi-frequency sonication Ultrason. Sonochem. 9 231-6

[19] Suo D, Govind B, Zhang S and Jing Y 2018 Numerical investigation of the inertial cavitation threshold under multi-frequency ultrasound Ultrason. Sonochem. 41 $419-26$.

[20] Gilles B, Béra J C, Mestas J L and Cathignol D 2006 Reduction of ultrasound inertial cavitation threshold using bi-frequency excitation Appl. Phys. Lett. 89 094106

[21] Saletes I, Gilles B, Auboiroux V and Bendridi N 2014 In vitro demonstration of focused ultrasound thrombolysis using bifrequency excitation BioMed Res. Int. 2014518787

[22] Mori E, Itoh K and Imamura A 1977 Analysis of a short column vibrator by apparent elasticity method and its applications Ultrasonics International 1977 Conference Proceedings p. 262

[23] Lin S 2007 Coupled vibration of isotropic metal hollow cylinders with large geometrical dimensions J. Sound Vib. 305 308-16

[24] Sherrit S, Dolgin B P and Bar-Cohen Y 1999 Modeling of Horns for Sonic/Ultrasonic Applications IEEE Ultrasonics Symp. pp 647-51

[25] Martin G E 1964 Vibrations of coaxially segmented, longitudinally polarized ferroelectric tubes J. Acoust. Soc. Am. 36 1496-506 